\title{
Construction and Evaluation of a Multipurpose Performance Check Phantom for Computed Tomography
}

\author{
L.E. Lubis ${ }^{1,4^{*}}$, I. Hariyati ${ }^{2}$, D. Ryangga ${ }^{3}$, I.A.S. Mu'minah ${ }^{4}$, T. Mart ${ }^{1^{*}}$ and D.S. Soejoko ${ }^{1,4}$ \\ ${ }^{1}$ Department of Physics, Faculty of Mathematics and Natural Sciences, Universitas Indonesia, Kampus UI, Depok 16424, Indonesia \\ ${ }^{2}$ Radiology Department, Gading Pluit Hospital, Jl. Boulevard Timur, Pegangsaan Dua, Jakarta 14250, Indonesia \\ ${ }^{3}$ Radiotherapy Department, Regional General Hospital, Jl. TB Simatupang No.1, Ps. Minggu, Jakarta 12550, Indonesia \\ ${ }^{4}$ Center for Medical Physics and Biophysics, Institute of Applied Sciences, Faculty of Mathematics and Natural Sciences, \\ Universitas Indonesia, Kampus UI, Depok 16424, Indonesia
}

\section{ARTICLE INFO}

Article history:

Received 10 November 2019

Received in revised form 30 March 2020

Accepted 30 March 2020

Keywords:

Computed tomography

Dose

Image quality

Phantom

\begin{abstract}
A B S T R A C T
The use of computed tomography (CT) has become a common practice in medical diagnosis in Indonesia. Its number, however, is not matched by the availability of dedicated-performance-check phantoms. This paper aims to describe the design, construction, and evaluation of an in-house phantom for CT performance check that accommodates both radiation dose measurement and image quality performance checks. The phantom is designed as laser-cut polymethyl methacrylate (PMMA) slabs glued together to form a standard cylindrical shape, with spaces to place dose measurement and image quality modules. In this paper, measurement results on both aspects are discussed and compared with standard phantoms and other works. For dose measurement, the constructed phantom exhibited the greatest absolute discrepancy against the reference standard phantom of $8.89 \%$. Measurement of the CT number linearity and modulation transfer function (MTF) yielded, at most, $7.51 \%$ and $5.07 \%$ discrepancies against Catphan 604, respectively. Meanwhile, although found to be more linear in the phantom-based contrast linearity test, the use of the in-house phantom for clinical image contrast threshold determination requires further study. For noise power spectrum (NPS) measurement, accurate results were obtained within a limited range of spatial frequency.
\end{abstract}

\section{INTRODUCTION}

The use of computed tomography (CT) as a medical imaging modality is increasingly prevalent worldwide. In Indonesia, the official facility repository of the Indonesian Ministry of Health (ASPAK system) recorded that $634 \mathrm{CT}$ scanners were in use per June 2019. Under several national regulations, it is compulsory that the performance of these CT devices be checked regularly both locally by clinical medical physicists and in external audit by the regulatory body under formal appointment to compliance tester companies. The regular performance check, which includes radiation dose measurement and image quality assessment, requires a set of tools, including phantoms, as standard patient substitute.

\footnotetext{
*Corresponding author.

E-mail address: lukmanda.evan@sci.ui.ac.id, terry.mart@sci.ui.ac.id DOI: https://doi.org/10.17146/aij.2020.1004
}

(C) 2020 Atom Indonesia. All rights reserved

Dose measurement is performed by using standard poly(methyl methacrylate) (PMMA) phantom with strictly prescribed materials and dimensions [1,2], while image quality assessment can be performed with a range of methods and phantoms available on the market [3,4]. Typical image quality assessment tools are available as cylindrical phantom with a number of modules to be scanned for test that consist of CT number linearity evaluation, low contrast assessment, and modulation transfer function (MTF) evaluation using small beads $[5,6]$.

While these tools are essential in ensuring CT performance, fewer than ten out of more than 2800 hospitals throughout Indonesia are currently equipped with the $\mathrm{CT}$ dose and image quality phantoms. Moreover, the number of compliance tester companies equipped with CT performance check tools was only fifteen in 2019. This implies a high importance of simple tools being made 
available for CT performance check purposes. The situation has been made urgent by the increase in number of CT scanners and sophisticated technology in use. With two separate sets of commercial phantoms for dose and image quality assessment being too costly to procure, there is an urgent need of multipurpose performance check phantoms.

This paper aims to describe the design and construction details of a new phantom specifically designed to address the lack of CT dose and image quality phantoms. The in-house phantom is designed as a multipurpose phantom with ability to serve as both dosimetry and image quality check in one tool. In addition, the result of measurement comparison with standard phantom is presented to demonstrate the projected performance of the new phantom as a preliminary evaluation.

\section{MATERIALS AND METHODS}

\section{Phantom material and design}

The IEC Standard 6061-2-44 dictates that CT dosimetry phantoms are made of polymethyl methacrylate (PMMA) [1]. Therefore, the proposed phantom is designed as PMMA stacks comprising a solid PMMA cylinder as shown in Fig. 1. All dimensions strictly follow standard head dosimetry phantom [1]. Twenty pieces of 1-cm-thick PMMA slabs (Karya Anugerah Kreasindo, Bekasi, Indonesia) were laser-cut using a $\mathrm{CNC}$ machine according to standard sizes and UV-glued together to form solid cylinder with rectangular space in the middle to accommodate inserts. No air gaps were observed between the stacks.
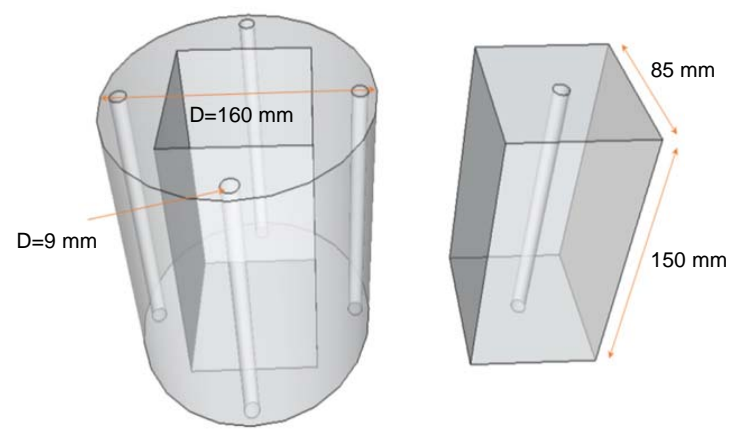

Fig. 1. Core phantom (left) and dosimetry insert (right).

Four insert objects (one dosimetry insert and three image quality inserts) are designed to be positioned inside the core phantom, filling the $8.5 \mathrm{~cm} \times 8.5 \mathrm{~cm}$ square space. For dosimetry use, the dosimetry insert is positioned inside the core phantom, and for image quality evaluation purposes, the three image quality inserts (Fig. 2) can be stacked and positioned accordingly.
The image quality insert for electron density evaluation provides eight materials mimicking water, PMMA, bone, lung (air), adipose, liver, muscle/grey brain matter, and white brain matter fabricated using organic materials with varied compositions. Physical test results regarding volumetric density, CT number, attenuation coefficients, and effective atomic numbers of these materials have been submitted for publication elsewhere and are outside the scope of this paper. All objects are $15 \mathrm{~mm}$ in diameter. Contrast resolution insert contains four groups of holes with diameters of $10 \mathrm{~mm}, 8 \mathrm{~mm}, 6 \mathrm{~mm}, 4 \mathrm{~mm}$, and $2 \mathrm{~mm}$. Each group was filled with cast resin mixed with $0.25 \mathrm{ml}, 0.50 \mathrm{ml}, 0.75 \mathrm{ml}$, and $1.0 \mathrm{ml}$ of iodine contrast agents (Iohexol, $350 \mathrm{mg} / \mathrm{ml}$ concentration) to produce four different contrast levels in each object sizes.

The MTF insert contains a copper wire of $0.19 \mathrm{~mm}$ diameter at the center of cast resin. The three image quality inserts can be positioned inside the core phantom with no specific sequence.

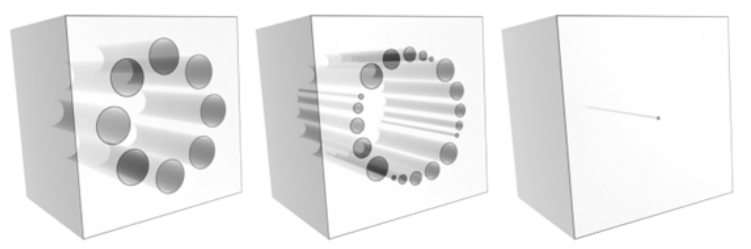

Fig. 2. Image quality inserts for (left) electron density evaluation, (center) contrast resolution assessment, and (right) MTF measurement.

\section{Phantom testing}

Both the dose and image quality measurement capability of the in-house phantom were evaluated. Dose measurement results were compared with a standard CTDI head phantom (Fig. 3) using a calibrated Radcal 10X6-3CT ionization chamber (Radcal, Monrovia, USA), while measured image quality aspects were compared with Catphan 604 (Phantom Laboratories, Salem, USA).

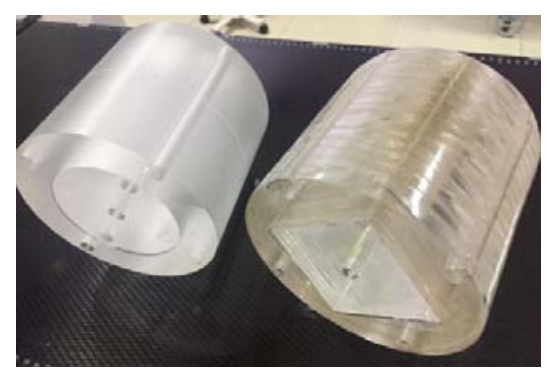

Fig. 3. Standard CTDI phantom (left) and constructed in-house phantom (right). 
Both tests were conducted on a Toshiba Aquilion 64 (Toshiba Medical System Corp., Ōtawara, Japan) operated in Radiotherapy Department, Pasar Minggu Regional General Hospital, Jakarta. The CT device used in this study had passed annual calibration test and compliance test.

For dose measurement capability test, air kerma in five standard measurement positions, i.e., center position, 12 o'clock, 3 o'clock, 6 o'clock, and 9 o'clock, were compared with measurement results on the standard phantom. Exposures were perfomed at $120 \mathrm{kVp}$ tube voltage, $200 \mathrm{~mA}$ tube current, $1 \mathrm{~s}$ gantry rotation time, and $8 \mathrm{~mm}$ beam collimation. These parameters comply with the CTDI measurement part of the compliance test protocol for CT scanners issued by the Indonesian Nuclear Energy Regulatory Agency (BAPETEN).

The electron density linearity, contrast linearity, and MTF were measured in both the inhouse phantom and the reference Catphan 604 phantom. Evaluation was performed quantitatively by comparing linear regression coefficients from the electron density evaluation and contrast resolution assessment inserts, as well as the MTF values between those obtained from in-house phantom and Catphan. The CT number accuracy comparison could not be performed since the materials used in the two phantoms differs; in the Catphan 604, the materials used are Teflon, Bone $50 \%$, Delrin, Bone $20 \%$, acrylic, polystyrene, low density polyethylene (LDPE), and polymethylpentene (PMP). Therefore, the linearity of the CT number was compared instead.

The linear regression coefficients for the electron density module were obtained by measuring individual pixel values from the objects and plotting them arbitratily. Meanwhile, a similar approach was performed for contrast resolution assessment, with an addition that the plotted data being signaldifference-to-noise ratio (SDNR) calculated using Eq. (1) $[7,8]$

$$
S D N R_{i}=\frac{N_{L}-N_{O, i}}{\sqrt{\frac{\left(S D_{O, i}{ }^{2}+S D_{L}^{2}\right)}{2}}}
$$

In (1), $\mathrm{SDNR}_{i}$ denotes the signal-difference-to-noise ratio of object $i ; N_{O, i}$ represents the mean pixel value of the object $i ; N_{L}$ indicates the mean pixel value of the background area adjacent to the object; $S D_{O, i}$ represents the standard deviation of the pixel value of the object $i$; and $S D_{L}$ denotes the standard deviation of the pixel value of the background.

The evaluation of the phantom's ability to serve as a tool for advanced system quality metric was performed by carrying out measurement of noise power spectrum (NPS) as a metric describing the distribution of noise magnitude in frequency domain [9-11]. The measurement was performed with the imQuest software package from Duke University $[12,13]$ and was compared with the results from other work using a similar type of standard phantom [11].

\section{RESULTS AND DISCUSSION \\ Dose measurement reading}

Figure 4 shows the dose measurement result from the use of the two phantoms. In general, measurement in all positions does not demonstrate major discrepancy. The in-house phantom exhibited its greatest absolute discrepancy from the reference standard phantom of $8.89 \%$ and its least discrepancy of $1.12 \%$ at the 3 'o clock and 6 o'clock positions, respectively. Individual discrepancies are shown in Table 1.

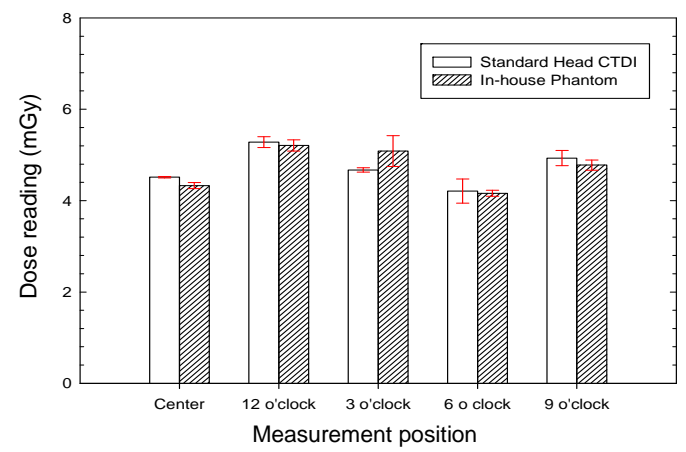

Fig. 4. Dose reading from in-house phantom comparison with standard CTDI phantom.

Table 1. Dose measurement result.

\begin{tabular}{|c|c|c|c|}
\hline \multirow{2}{*}{$\begin{array}{l}\text { Measurement } \\
\text { position }\end{array}$} & \multicolumn{2}{|c|}{ Air kerma reading (mGy) } & \multirow{2}{*}{$\begin{array}{c}\text { Mean } \\
\text { discrepancy } \\
(\%)\end{array}$} \\
\hline & $\begin{array}{c}\text { Standard } \\
\text { CTDI }\end{array}$ & $\begin{array}{l}\text { In-house } \\
\text { phantom }\end{array}$ & \\
\hline Center & $4.51 \pm 0.02$ & $4.33 \pm 0.07$ & 4.01 \\
\hline 12 o'clock & $5.28 \pm 0.12$ & $5.21 \pm 0.12$ & 1.38 \\
\hline 3 o'clock & $4.67 \pm 0.05$ & $5.08 \pm 0.34$ & 8.89 \\
\hline 6 o'clock & $4.21 \pm 0.26$ & $4.16 \pm 0.07$ & 1.12 \\
\hline 9 o'clock & $4.93 \pm 0.17$ & $4.78 \pm 0.11$ & 3.13 \\
\hline
\end{tabular}

The national compliance test regulation issued by BAPETEN dictates that the dose measurement results should not differ by $20 \%$ in order for a CT device to be certified. This difference refers to the discrepancy between measured and consoledisplayed $\mathrm{CTDI}_{\mathrm{vol}}$ values. The fact that the constructed phantom differs only by less than $10 \%$ from a standard phantom indicates that the constructed phantom can be used for dose measurement purposes without a doubt that the results may alter the decision on the CT scanner's state of performance. 


\section{Image quality metric evaluation}

The typical resulting image of the two phantoms are shown in Fig. 5. Since the initial aim of the phantom construction was not to create an identical phantom with the commercially-available tool, tissue-mimicking objects on the electron density module were not the same as in the Catphan phantom. Therefore, the first evaluation was performed to check the linearity of the CT number a parameter selected to represent electron density. Linearity is assessed by means of the R-squared values of the CT number. A similar approach was followed for contrast linearity by means of the SDNR.

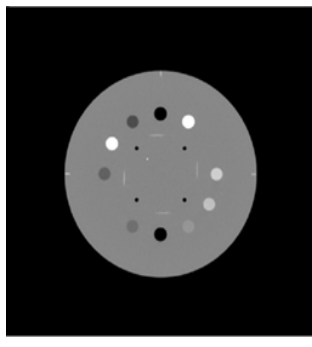

a

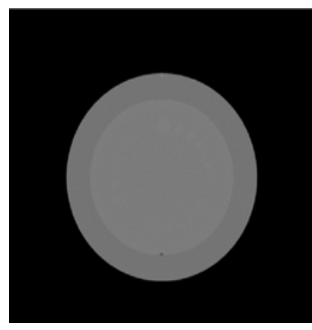

c

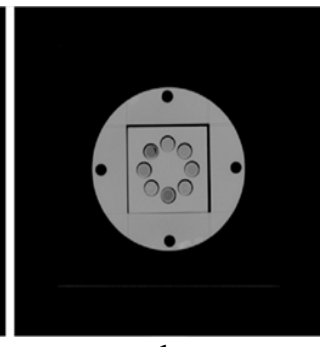

b

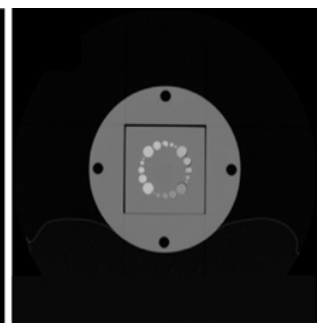

d
Fig. 5. Typical resulting images of electron density linearity ( $a$ and b) and contrast linearity checks ( $c$ and d). Images a and c are obtained from Catphan 604, while images $b$ and $d$ are obtained from in house phantom at $100 \mathrm{kVp}, 200 \mathrm{mAs}$. Images a and $\mathrm{b}$ are displayed with WL 10 and WW 1070, while images c and d are displayed with WL 200 and WW 2500.

Figure 6 shows CT number linearity measurement result on both the Catphan and the constructed phantom. The in-house phantom was $4.66 \%, 5.56 \%$, and $7.51 \%$ less linear than the Catphan at $80 \mathrm{kVp}, 100 \mathrm{kVp}$, and $120 \mathrm{kVp}$, respectively. This is mainly caused by the presence of two water- and adipose-mimicking objects originating from different raw materials (i.e., flourbased, FB, and carbon-based, CB). These two materials have demonstrated a difference in $\mathrm{CT}$ number and therefore are included in this study for linearity check. The result of contrast linearity measurement is shown in Fig. 7. While the constructed phantom does not demonstrate major contrast linearity difference from Catphan 604, a major difference is observed in the contrast magnitude.

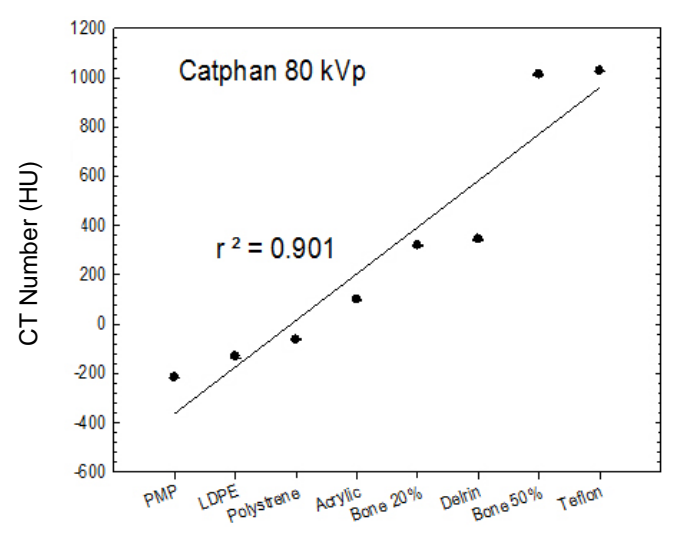

$6 \mathrm{a}$

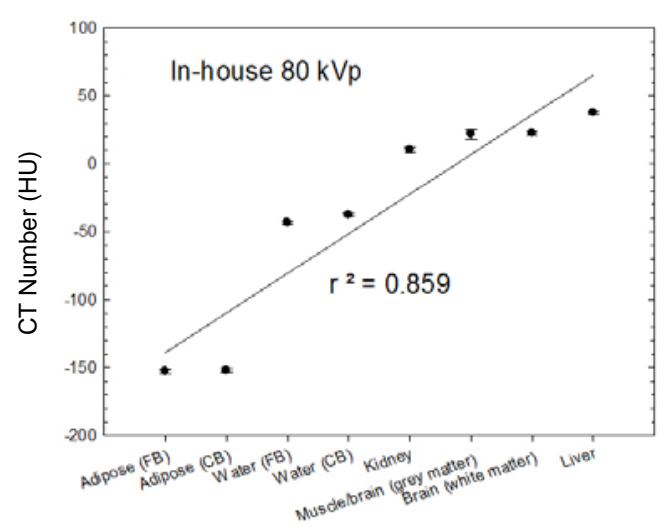

$6 \mathrm{~b}$

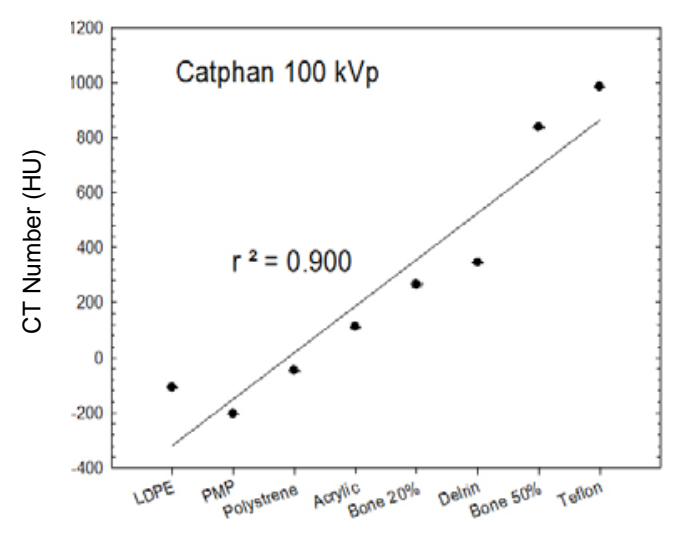

$6 \mathrm{c}$

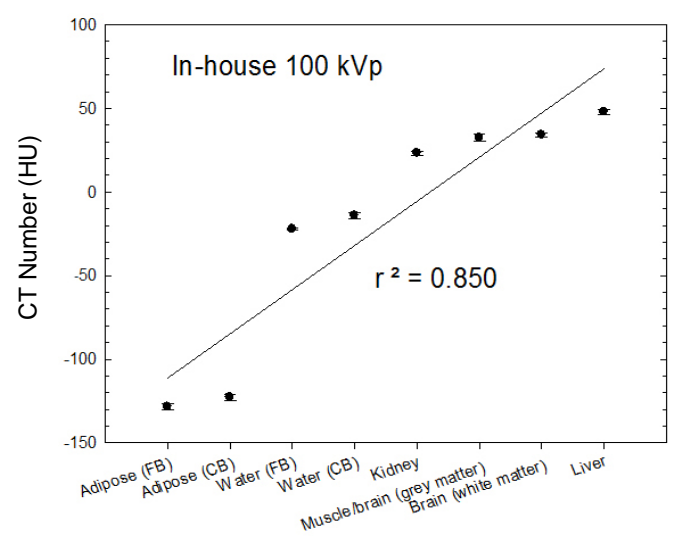

$6 d$ 


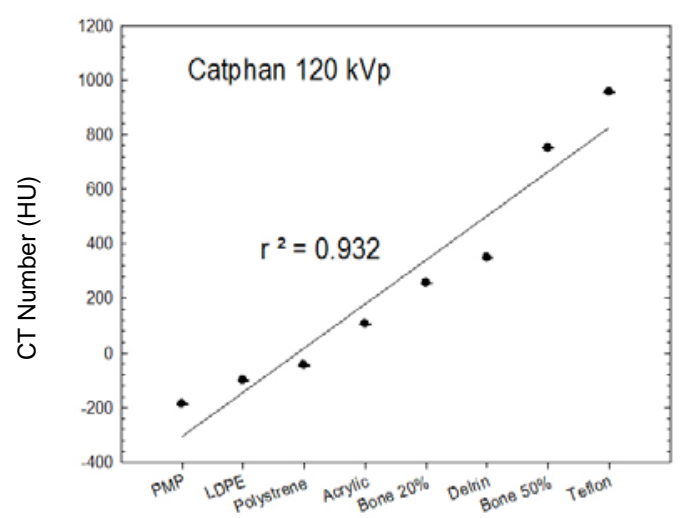

$6 \mathrm{e}$

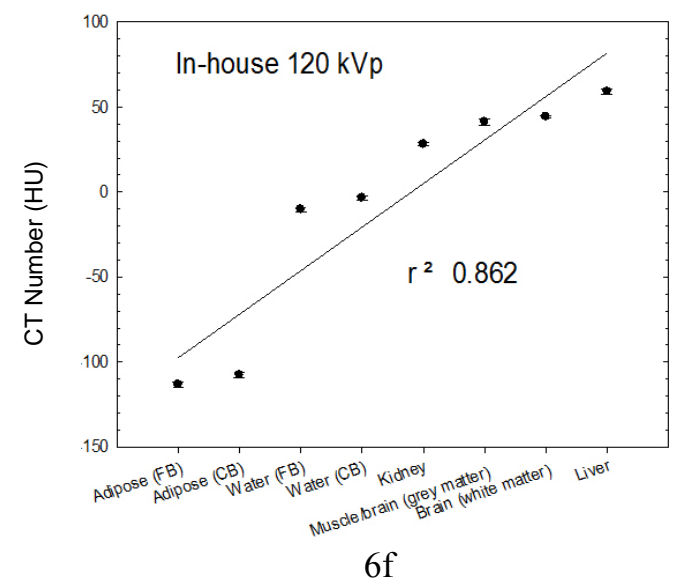

Fig. 6. CT number linearity measurement result for image a, c and e Catphan 604 and image $\mathrm{b}, \mathrm{d}$ and $\mathrm{f}$ constructed phantom at $80 \mathrm{kVp}$ image a and b, $100 \mathrm{kVp}$ image $\mathrm{c}$ and $\mathrm{d}$, and $120 \mathrm{kVp}$ image e and $\mathrm{f}$.
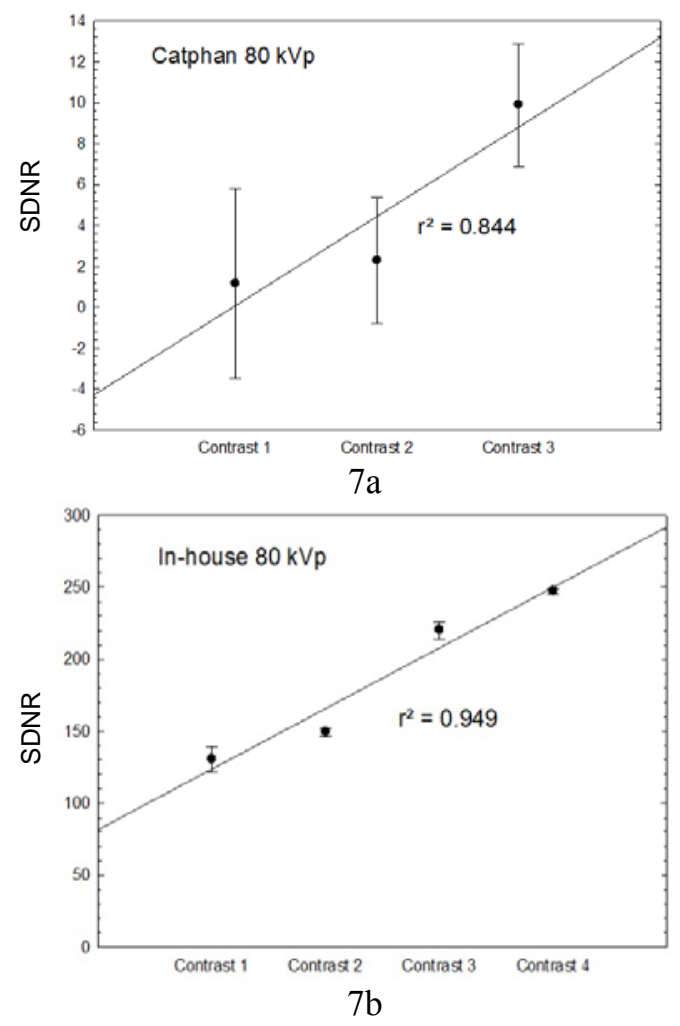
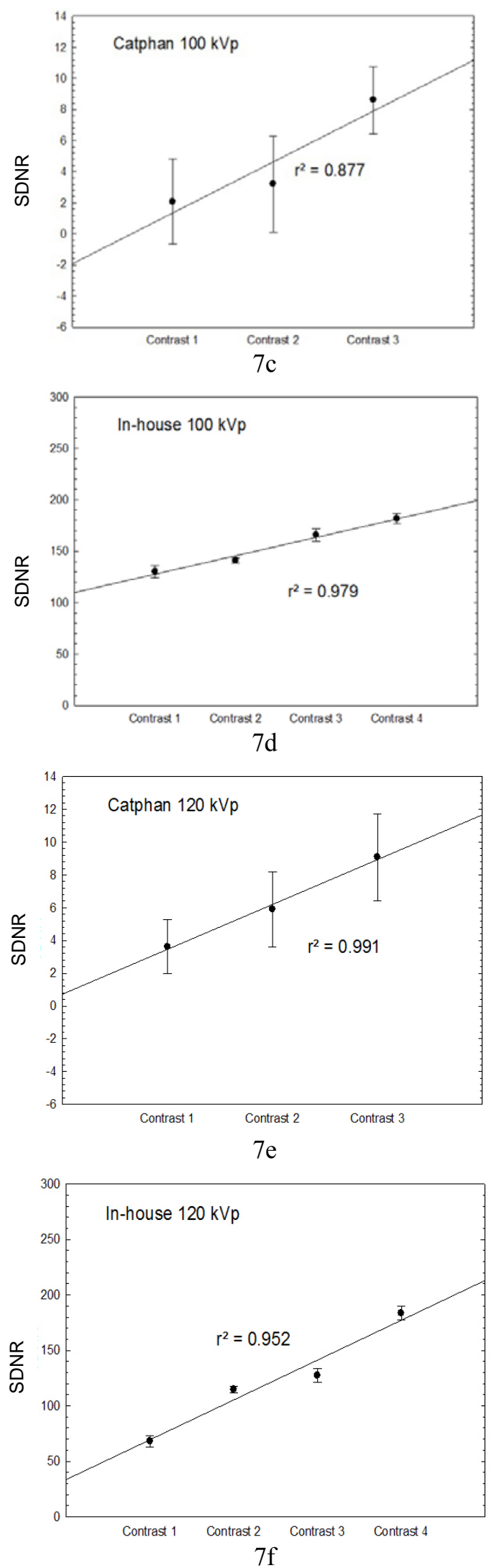

Fig. 7. Contrast linearity measurement result for image a, c and e Catphan 604 and image $\mathrm{b}, \mathrm{d}$ and $\mathrm{f}$ constructed phantom at $80 \mathrm{kVp}$ image a and b, $100 \mathrm{kVp}$ image $\mathrm{c}$ and $\mathrm{d}$, and $120 \mathrm{kVp}$ image e and $f$. 
The Rose contrast model suggests that there is a human eye visibility threshold at SDNR $=5$ [14]. The contrast level presented by the constructed phantom highly exceeds the aforementioned threshold by a factor of ten compared to Catphan 604. It implies that the phantom module, produced with a minimum volume of iodine contrast agent, provides higher detectability for assessment of contrast threshold and visibility. The result also suggests that the use of iodine contrast agent as solvent in resin provides superior detectability, even when minimally used. Furthermore, the correlation of phantom-based object contrast with clinical image contrast visibility requires further investigation.

In Fig. 8, it can be deduced that the thin wire on the constructed phantom can be used to measure MTF with the result being $2.00 \%, 4.45 \%$, and $5.07 \%$ higher than Catphan 604 at $80 \mathrm{kVp}$, $100 \mathrm{kVp}$, and $120 \mathrm{kVp}$, respectively. Theoretically, MTF is not under the influence of exposure parameters. This is confirmed by the result using inhouse phantom.

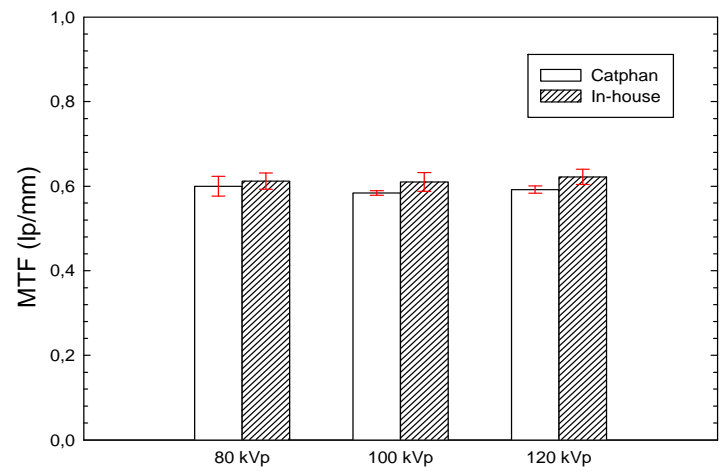

Fig. 8. Modulation transfer function measurement results from varying tube voltages on Catphan 604 and the in-house phantom

The NPS comparison between the in-house phantom and the standard phantom as used by Winslow et al. (2017) [11] and Ria et al. (2018) [15] is presented in Fig. 9. While the average relative deviation from the reference phantom was found to be $22 \%$, the peak noise frequency deviated by $0.05 \mathrm{~mm}^{-1}$, while the average noise frequency was identical. Furthermore, the noise magnitude differed by merely 0.6 Hounsfield units (HU). Particular interest is drawn to the spectral shapes, where it is observed that the in-house phantom is able to completely reproduce the result from reference method at low spatial frequencies $\left(<0.1 \mathrm{~mm}^{-1}\right)$. This result encouraged the use of the in-house phantom to quantify noise level in homogenuous objects.

Based on the findings on dose measurement and image quality metric quantification capability, the use of the in-house phantom in assessing CT system performance can be suggested for routine use by clinical medical physicists. The designed phantom's capability to serve as dose measurement tool allows routine application for dose survey in the clinical setting [16], establishment of diagnostic reference levels [17], and dose comparison upon introduction of new techniques or technology [18]. Contrast and CT number linearity reproducibility also indicated that the in-house phantom can also be useful for image quality quantification as well as dose management and optimization studies [19,20]. The use of the in-house phantom for measuring advanced operational performance metrics, such as NPS, however, would require design optimization to enable the phantom to measure NPS with comparable sensitivity with standard homogeneous phantoms.

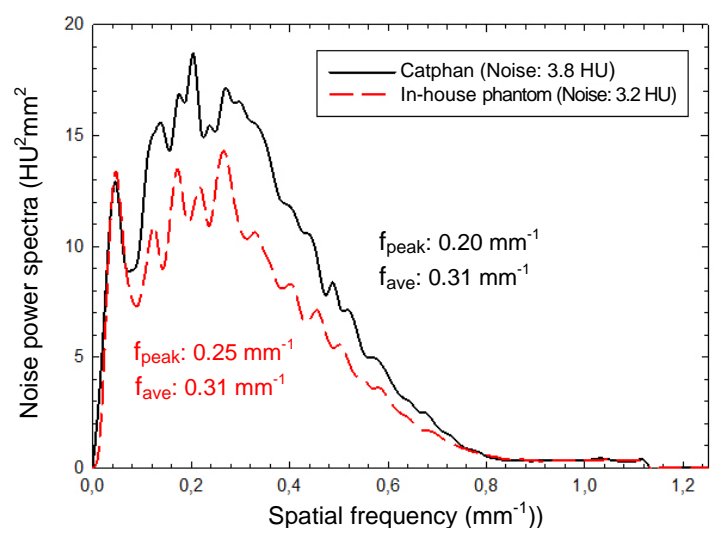

Fig. 9. Noise power spectrum measurement results on Catphan 604 and the in-house phantom under identical energy.

\section{CONCLUSION}

The designed and constructed phantom can be suggested for routine use in terms of head CTDI measurement, CT number linearity assessment, and MTF measurement. Furthermore, the measurement result on the contrast linearity module, in addition to indicating conformity with Catphan 604, also suggests that the use of iodine contrast agent as solvent in resin provides superior phantom object detectability. Despite being able to measure advanced image quality metric such as NPS with relatively accurate quantitative results, the in-house phantom's sensitivity over the appropriate spatial frequency range needs improvements.

\section{ACKNOWLEDGEMENT}

This work was partially funded by Universitas Indonesia's Indexed International Publication Grant 2018 (Hibah PITTA UI 2018) with contract number 2278/UN2.R3.1/HKP.05.00/2018. The authors would also like to thank the Radiotherapy 
Department, Pasar Minggu Regional General Hospital, for facilitating the use of its CT device.

\section{REFERENCES}

1. Anonymous, International Electrotechnical Commission, IEC 60601-2-44 (2009).

2. H.D.H. Gala, A. Torresin, A. Dasu et al., Phys. Medica 39 (2017) 67.

3. F.R. Verdun, D. Racine, J.G. Ott et al., Phys. Medica 31 (2015) 823.

4. T. Takenaga, S. Katsuragawa, M. Goto et al., Radiol. Phys. Technol. 8 (2015) 53.

5. P. Monnin, A. Viry, F.R. Verdun et al., Phys. Med. Biol. 65 (2020) 1.

6. C. Lee, H.D. Song and J. Baek, Med. Phys. 47 (2020) 2838

7. L.E. Lubis, I. Bayuadi, S.A. Pawiro et al., Phys. Medica 31 (2015) 659.

8. L.E. Lubis, L.A. Craig, H. Bosmans et al., Phys. Medica 46 (2018) 114.

9. E. Samei, D. Bakalyar, K.L. Boedeker et al., Med. Phys. 46 (2019) e735.
10. E. Samei, D. Bakalyar, K. Boedeker et al., Performace Evaluation of Computed Tomography Systems, AAPM Report No. 233 (2019).

11. J. Winslow, Y. Zhang and E. Samei, Med. Phys. 44 (2017) 5705.

12. F. Ria, J.B. Solomon, J.M. Wilson et al., Med. Phys.47 (2020) 1633.

13. J. Solomon, Y. Zhang, J. Wilson et al., Med. Phys. 45 (2018) E134.

14. A.E. Burgess, J. Opt. Soc. Am. A 16 (1999) 633.

15. F. Ria, J. Solomon, Y. Zhang et al., Med. Phys. 45 (2018) E136.

16. A. Mokhtar, M. Elawdy, M.A. El-Hamid et al., Egypt. J. Radiol. Nucl. Med. 48 (2017) 701.

17. D.H. Salama, J. Vassileva, G. Mahdaly et al., Phys. Medica 39 (2017) 16.

18. R.E. Moorin, D.A.J. Gibson, R.K. Forsyth et al., PLoS One 10 (2015) e0138329.

19. A. Parakh, M. Kortesniemi and S.T. Schindera, Radiology 280 (2016) 663.

20. H. Dang, J.W. Stayman, J. Xu et al., Phys. Med. Biol. 62 (2017) 8693. 